\title{
Building Inertial Electrostatic Confinement Fusion Device Aimed for a Small Neutron Source
}

\author{
Gamal M. El-Aragi \\ Plasma Physics and Nuclear Fusion Department, Egyptian Atomic Energy, Cairo, Egypt \\ Email address: \\ elaragi@gmail.com \\ To cite this article: \\ Gamal M. El-Aragi. Building Inertial Electrostatic Confinement Fusion Device Aimed for a Small Neutron Source. International Journal of \\ High Energy Physics. Vol. 4, No. 6, 2017, pp. 88-92. doi: 10.11648/j.ijhep.20170406.13
}

Received: October 20, 2017; Accepted: November 1, 2017; Published: December 15, 2017

\begin{abstract}
The aim of this paper introduces the preliminary results of the design and construction of first Egyptian inertial electrostatic confinement IEC fusion device. It consists of $2.8 \mathrm{~cm}$ stainless steel cathode, $6.5 \mathrm{~cm}$ anode diameter with $10 \mathrm{~cm}$ diameter $30 \mathrm{~cm}$ height vacuum chamber. The operation of IEC experiments has concentrated on pulsed operation to achieve the high currents required to generate increased reactions rates. The discharge voltage waveform with peak voltage $20 \mathrm{kV}$ with a full width half maximum (FWHM) of 10 nanoseconds and current pulse waveform has been registered using pick-up coil with peak current about $150 \mathrm{~mA}$. Experiments are performed with air as operating gas at different pressures and voltages. Time resolved of $\mathrm{x}$-ray radiation signals are obtained using fast radiation detector.
\end{abstract}

Keywords: Electrostatic, Confinement, Fusion, X-ray

\section{Introduction}

An inertial electrostatic confinement is a device that uses an electric field to heat ions to conditions suitable for nuclear fusion. The machine generates an electric potential difference between two metal cages inside a vacuum. Positive ions fall down this voltage drop, building up speed. If they collide in the center, they can fuse. This is a type of inertial electrostatic confinement device [1]. Inertial Electrostatic Confinement (IEC) is a fusion concept in which fuel ions are trapped with purely electrostatic fields in a convergent geometry, as opposed to magnetic confinement. It consists of a pairs of spherical electrodes in which the central one (cathode) is negatively high-voltagebiased and the outward electrode (anode) is grounded [2]. The basic IEC approach is to create a potential well through electrostatic Confinement of one of the plasma species in a dynamic (inertial) configuration. Inertial effects associated with dynamic motion of the confined species are essential to avoid plasma losses predicted for systems by Earnshaw [3]. Credit for its invention is independently given to U. S. scientist Philo Farnsworth and Russian scientist Oleg Lavrent yev, as both formulated means of electrostatic confinement almost simultaneously throughout the 1950 and into the $1960[4,5,6,7]$.
If a negative voltage of some kilo-volts is brought upon it (an inner grid), a glow discharge starts if the gas pressure in the vessel is low enough. Electrons move towards the positive charged outer grid. At collisions with neutral gas atoms, also some positive ions are produced which immediately move towards the negative inner grid. If pressure is quite low there are few further collisions between electrons/ions and gas atoms. It can happen now an electron or ion travels all the distance between the grids in a straight line and becomes quite fast. Approaching the grid, most will slip through a mesh and continue to travel that direction. The objective for a power reactor is to obtain ion beam-beam collisions in this central core. for neutron/proton production, satisfactory reaction rates can come from beam-background collisions. Applications of high Q (where Q is the ratio of fusion power produced Pf to power consumed Pin) IEC reactors are obvious as they could be used for terrestrial energy generation and also as the main energy sources for space vehicles [8, 9]. Low Q IEC devices also have a substantial number of applications as they can produce a copious amount of neutrons for neutron detector calibration $[10,11]$.

A novel approach for improving the neutralization in ioninjected IEC devices involves the active injection and confinement of electrons to the center of the device, to create 
dense, neutralizing plasma in the core region. Enhanced neutralization allows for higher ion densities and populations, and consequently higher fusion rates [12].

\section{Experimental Set-up}

The IEC consists of a cylindrical vacuum chamber made of Pyrex glass tube with $10 \mathrm{~cm}$ of diameter and $30 \mathrm{~cm}$ height. The inner electrode consist of two stainless steel rings which are welded in their joint places (Figure 1) and spiral outer electrode made of stainless steel. Figure 1 displays schematic diagram of the construction details of the IEC fusion set-up. Table 1 shows some parameters of IEC fusion device.

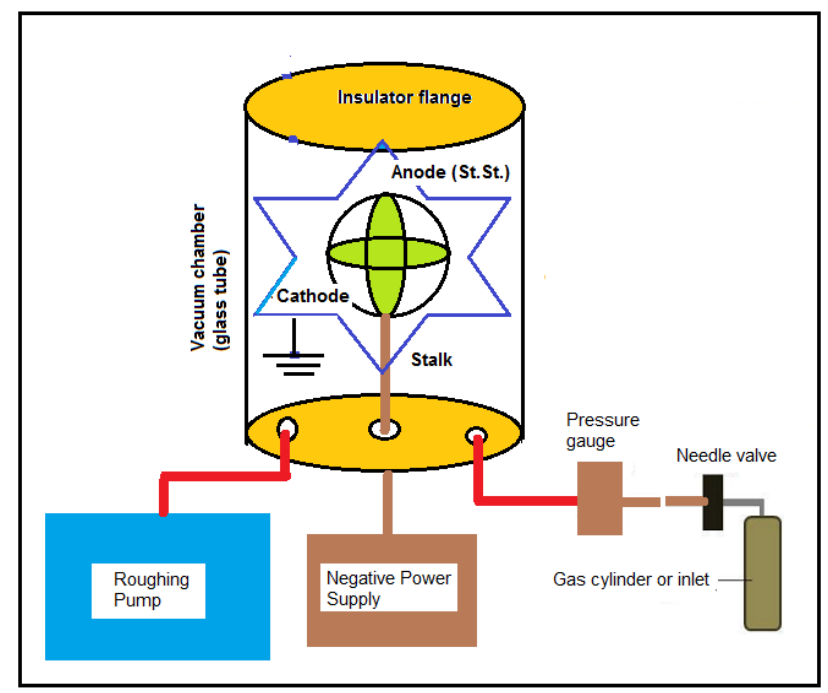

Figure 1. Scheme of experimental setup.

\section{Design High Voltage System}

$\mathrm{D}-\mathrm{D}$ fusion can occur in an IEC device at voltages as little as $10 \mathrm{kV}$ or less, but detectable fusion generally does not occur until voltages are at least $15 \mathrm{kV}$ [13]. Preassembled power supplies are available but expensive. For this project, a negative DC power supply was built.

Table 1. Design and operational parameters of IEC fusion device.

\begin{tabular}{ll}
\hline Parameter & Value \\
\hline Vacuum chamber diameter & $10 \mathrm{~cm}$ \\
Vacuum chamber height & $30 \mathrm{~cm}$ \\
Anode grid diameter & $6.5 \mathrm{~cm}$ \\
Cathode grid diameter & $2.8 \mathrm{~cm}$ \\
High-voltage Stalk height & $12 \mathrm{~cm}$ \\
Cathode voltage & $20-30 \mathrm{kV}$ \\
Air pressure & $0.001-0.1$ Torr \\
\hline
\end{tabular}

A power supply, transformer $20 \mathrm{kV}$ and high voltage diodes have been used for this purpose. During the first positive half-cycle, which is designated on the sine wave graph as $\mathrm{T} 1$, the voltage from the transformer increases accordingly with the polarity shown. The current flows in the direction of the arrows, charging the capacitor through the diode. During the capacitor charging time there is no voltage to the inner grid because the current takes the course of least resistance. In other words, rather than take a path through ground and up to the plate of the cathode, the current swings up through the diode. The voltage across the capacitor will rise to the transformer secondary voltage to the maximum $20 \mathrm{kV}$ volts. As the transformer secondary voltage begins to decrease from its maximum positive value (at time increment T2 on the sine wave graph), the capacitor will attempt to discharge back through the diode. The diode is like a oneway street in that it will not conduct in this direction. Thus, the discharge path is blocked, and the capacitor remains charged to the $20 \mathrm{kV}$ volts.

At time T3, the transformer secondary (output) voltage swings into the negative half-cycle and increases in a negative direction to a negative $20 \mathrm{kV}$ volt. The transformer secondary and the charged capacitor are now essentially two energy sources in series. The $20 \mathrm{kV}$ volts across the transformer winding add to the $20 \mathrm{kV}$ volts stored in the capacitor and the sum voltage of $40 \mathrm{kV}$ volts is applied to the cathode. There are two fundamental characteristics of this $40 \mathrm{kV}$-volt output that should be noted. First, because a voltage doubler is also a rectifier, the output is a DC voltage. Second, the resulting output voltage that is applied to the cathode is actually a pulsed DC voltage. This is because the doubler generates an output only during the negative halfcycle of the transformer's output (secondary) voltage. So, the inner grid is, in fact, pulsed on and off at a rate of 50 times per second. Thus the voltage doubler circuit has been used to conduct the spark gap (S. G.).
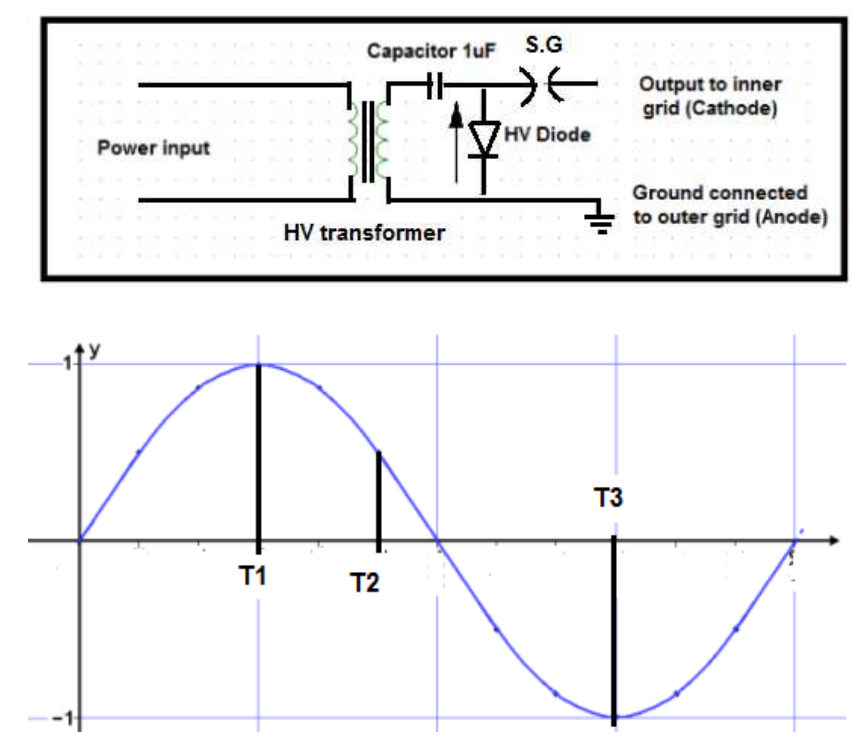

Figure 2. Circuit diagram of the power supply.

\section{Results and Discussion}

A small amount of air was admitted using the needle valve until pressure started to rise. The camera monitor and current indication was watched for expected plasma indications. When plasma was seen visually and through rising grid current, pressure was stabilized with the needle valve. Observations were recorded for plasma characteristics including color and shape. Stable combinations of current, 
pressure, and voltage were recorded. Voltage, current, and pressure were varied to determine effects on the plasma. The blue color glow is caused by electrons emitted from cathode (inner grid) in air gas produce it. Raising voltage intensified the light of the plasma and round with a bugle shaped light on one side. Further increases in voltage caused the plasma to shrink but intensify in brightness in the center (Figure 3). Understanding voltage and current was essential to reactor operation to monitor plasma status and grid health to prevent overheating

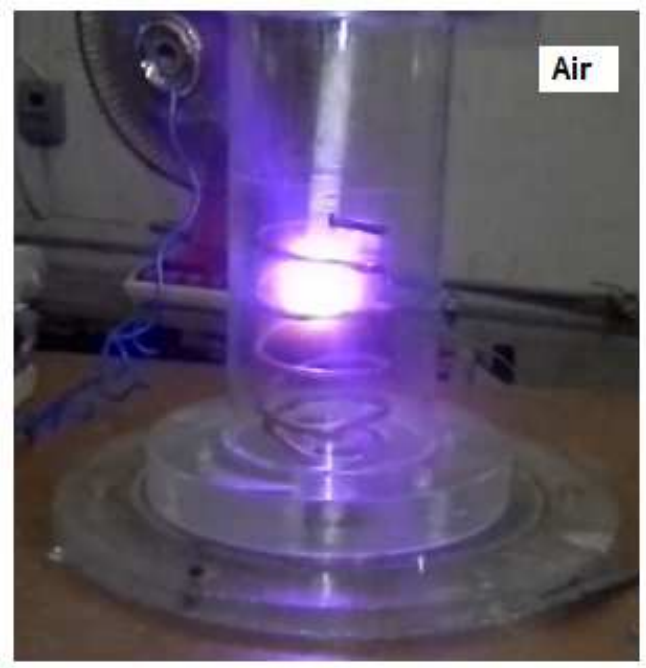

Figure 3. Glow discharge of air plasma.

The applied voltage to and the discharge current through the discharge chamber were measured using a voltage divider (homemade), which was connected between the two electrodes, and a current monitor, which can be located upon returning to the ground. The signals from the voltage divider and the current monitor were recorded in a digitizing oscilloscope (Lecroy, USA) with $200 \mathrm{MHz}$ bandwidth.

Figures 4 and 5 indicate the current and voltage waveforms characterizing the pulsed IEC fusion device.

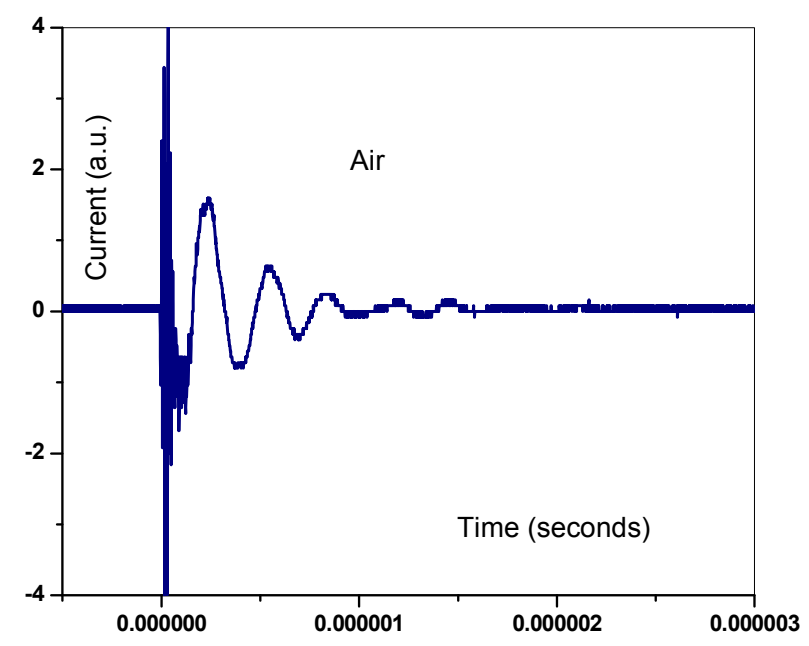

Figure 4. Discharge current signal from air plasma.

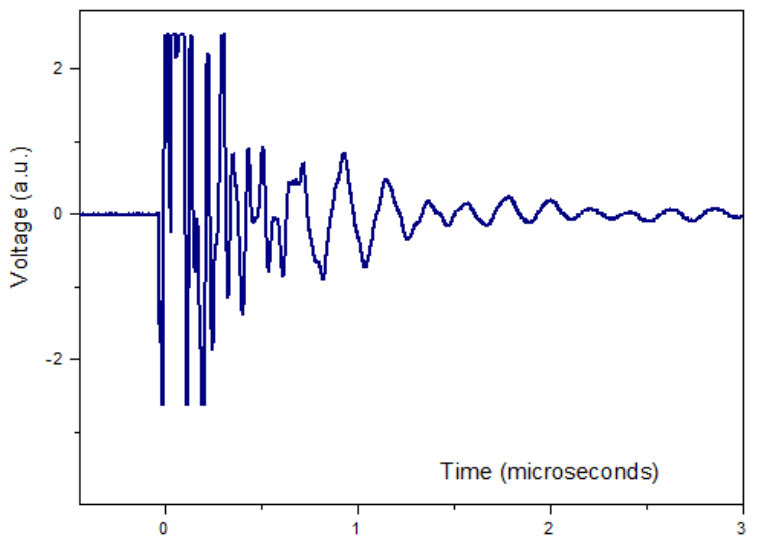

Figure 5. Discharge voltage signal from air plasma.

From these measurements, the power transfer into the IEC device can be calculated. The power was also obtained as a function of time as shown in figure 6 .

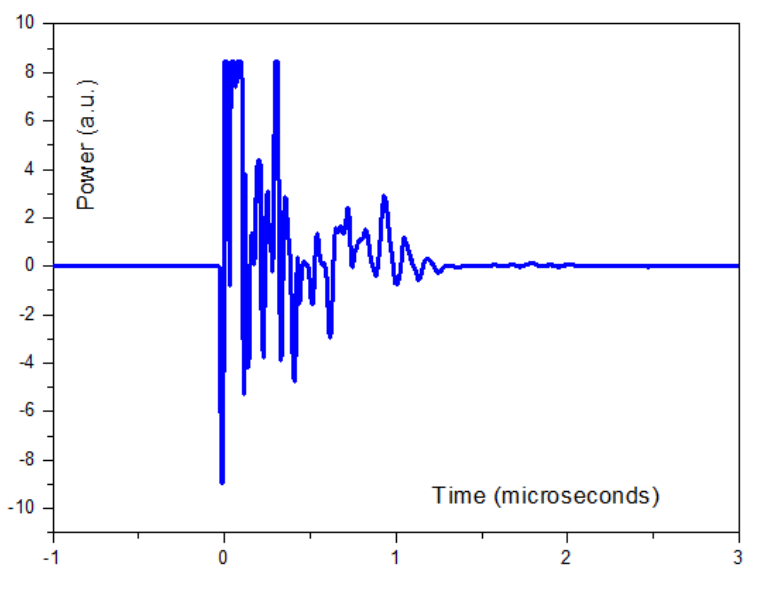

Figure 6. Time variation of the power.

Figure 7 shows the relation between discharge current, in $\mathrm{mA}$ versus the discharge voltage, in $\mathrm{kV}$ using air gas. It is clear that the discharge current increases by increasing the discharge voltage. At higher voltages more electrons are extracted from the cathode surface per unit area and the current increases.

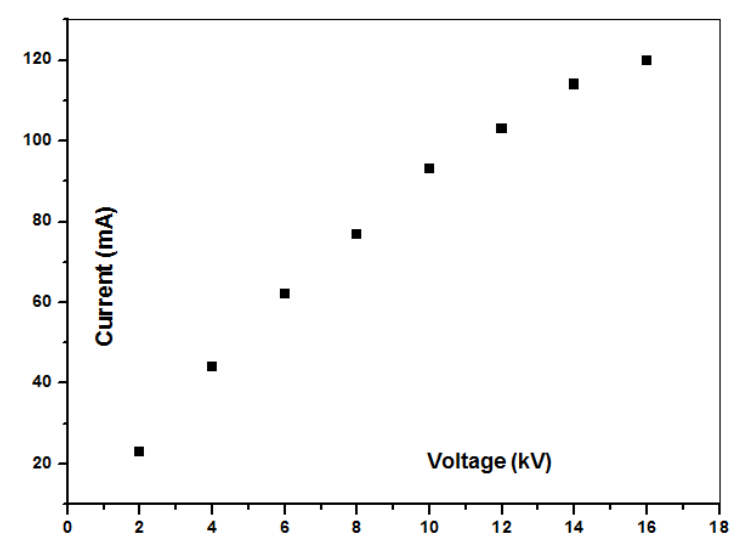

Figure 7. Relation between discharge voltage and discharge current for IEC plasma device. 
The generation of x-ray has been investigated experimentally in the IEC fusion reactor using fast radiation detectors based on type of photomultiplier with high anode characteristics, nanosecond time resolution, shielded against electromagnetic interference in the assembly with fast scintillator. In the development of the detector housings special attention is paid to the screening of the pulsed electromagnetic interference occurring at the time of discharge. X-ray yield in perpendicular direction have been registered by type of photomultiplier shown in figure 8 .

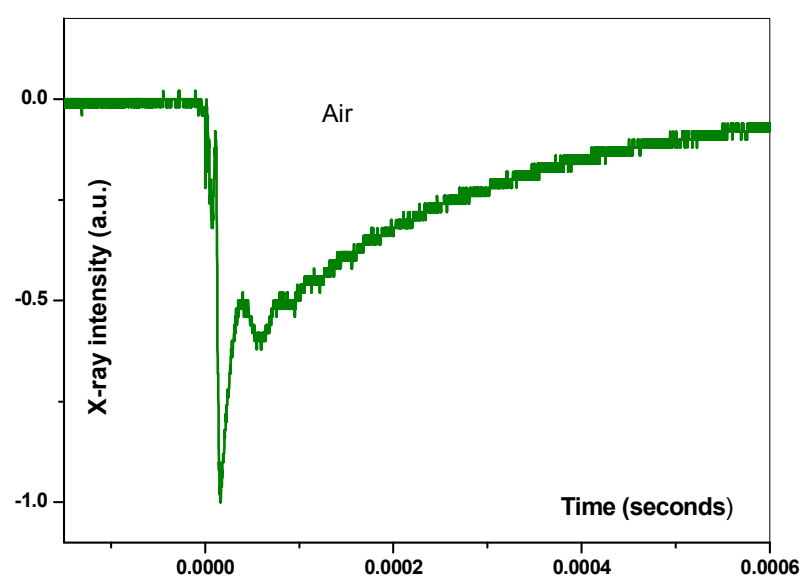

Figure 8. Oscilloscope trace taken by PMT placed near from the IEC chamber.

The measured X-ray intensity is plotted in figure 9 versus the cathode voltage for different pressures. The X-ray intensity increases with an increase of the cathode voltage at constant pressure, and with an increase of IEC chamber pressure at the constant voltage.

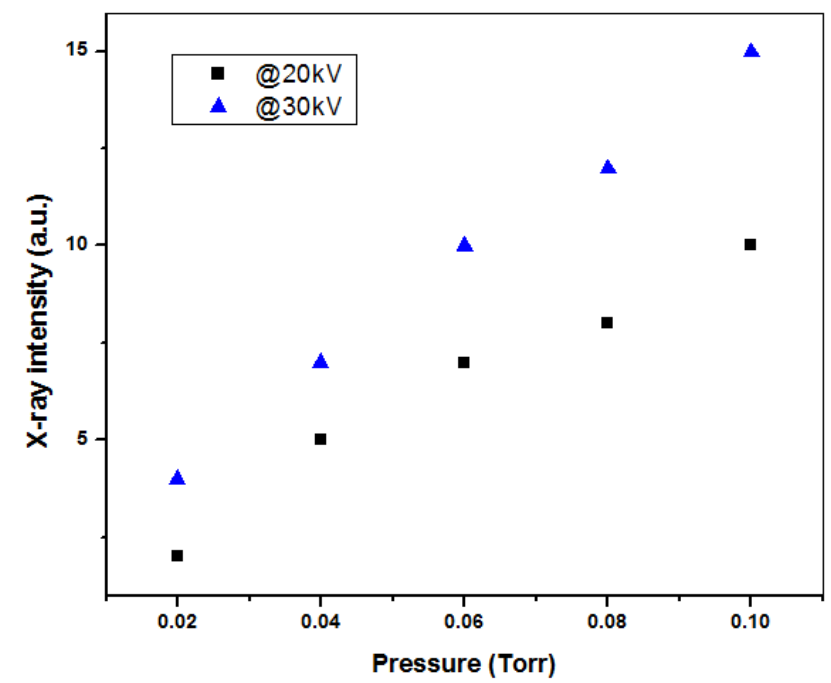

Figure 9. X-ray production as a function of pressure for different voltages.

Typical photomultiplier signal corresponds to plasma structures in the reactor for air gas can be observed (figure 10). The photomultiplier voltage trace include one pulse of interest is due to the primary discharge. Both intensity and duration of the pulses are different. This can be related to the different kinetics of elementary processes in the plasma structures. Although these results are obtained by measuring the total light emission from the plasma in the observation region, they represent valuable data of the plasma dynamics of fusion reactor. The change in the full width at half maximum (FWHM) and amplitude of each signal is due to the kinetic energy of particles entering and leaving the plasma source.

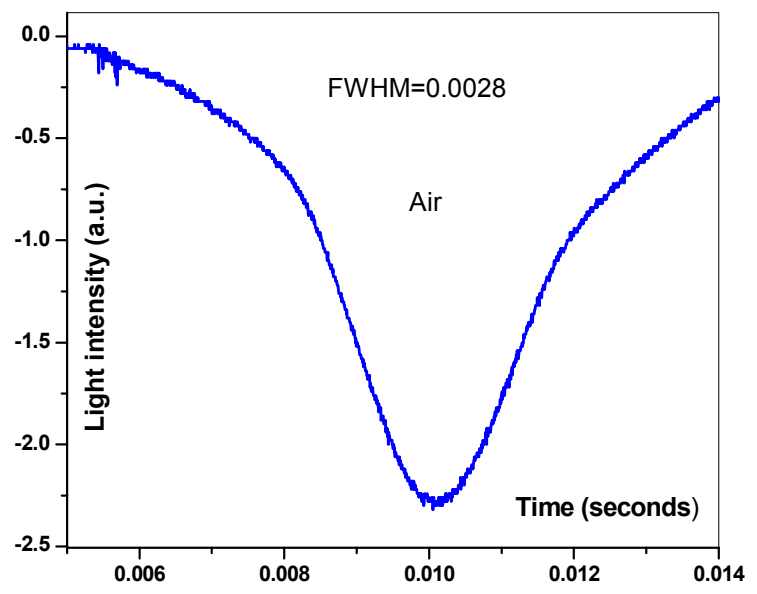

Figure 10. PMT signal of air plasma Source.

The Fluke 80TK is a thermocouple converter for use with any K-type thermocouple and a digital multimeter or oscilloscope. The 80TK accepts the output of any K-type thermocouple and converts it to $1 \mathrm{mV}$ per degree (Celsius or Fahrenheit) after calibration. Measurement Range: -50 to 1000 degrees Celsius or -58 to 1832 degrees Fahrenheit. Temperature is measured by exposing or attaching the thermocouple to the surface to be measured. The multimeter or oscilloscope displays the temperature directly in degrees Fahrenheit or Celsius. In this experiment, the thermocouple probe was placed at $2 \mathrm{~cm}$ above the cathode to monitor the temperature. This thermocouple probe picked up the maximum temperature in its focus. It has found that the temperature about $78^{\circ} \mathrm{C}$ after one minute as shown in figure 10 .

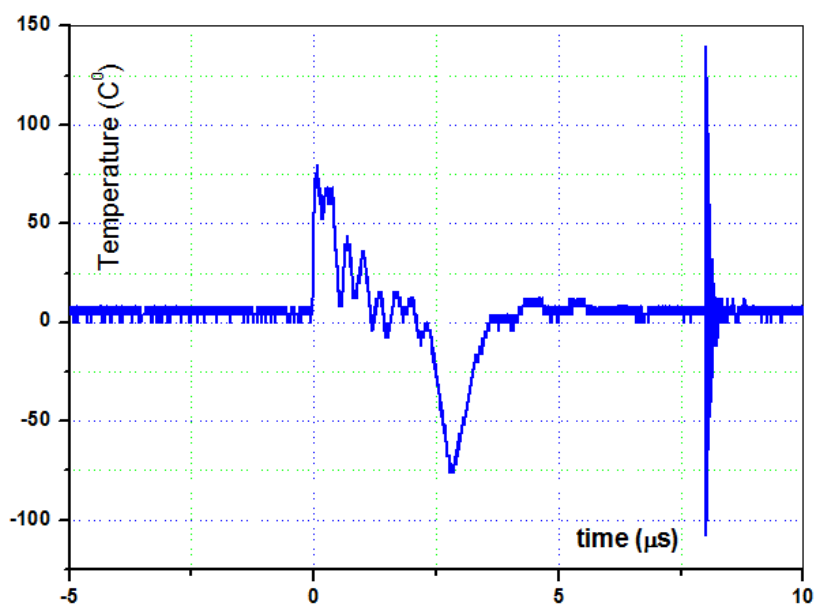

Figure 11. Oscilloscope trace of thermocouple probe. 


\section{Theoretical}

For using deuterium gas, the rate of DD-neutron generation is given by [14]

$$
\frac{d N}{d t}=\frac{1}{2} n_{i}^{2} \sigma\left(\epsilon_{i}\right) U_{i} \Omega
$$

Where $\Omega$ is volume corresponding to maximum ion density, $\sigma$ is cross-section of fusion reaction, $n_{i}$ is the final density of deuterium ions and $U_{i}$ is the speed of deuterium ion.

The final density of deuterium ions is given by

$$
n_{i} \approx 2.7 \times 10^{2} f \varphi^{1.5} v^{2} \mathrm{~cm}^{-3}
$$

Where $\varphi$ is the depth of potential well (the potential of the electric field in the axis of the system), $f \leq 1$ is the ratio of ion density to electron density and $v$ is the oscillation frequency is given by

$$
v \approx 8 \times 10^{6} \frac{\sqrt{\varphi}}{r_{g}} H z
$$

Where $r_{g}$ is the initial radius of inner cathode in $\mathrm{cm}$.

For $r_{\mathrm{g}}=1.4 \mathrm{~cm}, \varphi=20 \mathrm{kV}$ then the oscillation frequency equal $10 \mathrm{MHz}$ approximately and final density of deuterium ions equal $10^{16}-10^{18} \mathrm{~cm}^{-3}$ for $\varphi=20 \mathrm{kV}-40 \mathrm{kV}$.

Then the rate of DD-neutron generation $(\mathrm{dN} / \mathrm{dt})$ equal to $10^{6}-10^{8}$ neutrons/second

\section{Conclusion}

The IEC fusion device is a powerful source of $\mathrm{x}$-ray radiations which may be suitable for different applications. The same device that produce $\mathrm{x}$-ray yield can be used to generate neutrons yield. The calculated rate of DD-neutron generation using the same electrode configuration about $10^{6}$ $-10^{8}$ neutrons/second.

\section{References}

[1] blogspot.com - Will's Amateur Science and Engineering: Fusion Reactor's First Light!, Feb 2010 (from blog).
[2] D. R. Boris et al., Phys. Rev. E 80, 036408 (2009).

[3] Earnshaw S (1842) On the nature of the molecular forces which regulate the constitution of the luminiferous ether. Trans Camb Philos Soc 7: 97-112.

[4] P. T. Farnsworth, "Electric Discharge Device for Producing Interactions Between Nuclei,” U. S. Patent \#3,258,402, June 1966.

[5] P. T. Farnsworth, "Method and Apparatus for Producing Nuclear Fusion Reactions," U. S. Patent \# 3,386,883, June 1968.

[6] O. A. Lavrent'ev, "Investigation of an electromagnetic trap," AEC-tr-7002, (1970).

[7] O. A. Lavrent'ev, "Electrostatic and Electromagnetic HighTemperature Plasma Traps," Annals N. Y. Acad. Sci., vol. 251, pp. 152, 1975.

[8] G. H. Miley, H. Momota, "A collimator-converter system for IEC propulsion," Space Technology and Applications International Forum-STAIF 2002, 2002, pp. 768-779.

[9] C. C. Dietrich, "Improved Particle Confinement in Inertial Electrostatic Fusion for Spacecraft Power and Propulsion", Ph. D. Dissertation, Massachusetts Institute of Technology, Feb. 2007.

[10] J. H. Nadler, "Space-Charge Dynamics and Neutron Generation in an Inertial Electrostatic Confinement Device", Ph. D. Dissertation, University of Illinois Urbana-Champaign, 1992.

[11] B. Jurczyk, "Theory and development of a sealed DeuteriumTritium Inertial Electrostatic Confinement Neutron Generator," M. S. Thesis, University of Illinois UrbanaChampaign, 1997.

[12] Brendan Sporer "A method for Active Space Charge Neutraliztion in an Inertial Electrostatic Confinement(IEC) Nuclear Fusion on Device" Ph. D. Dissertation, The Pennsylvania State University, Spring 2017.

[13] Hull, Richard. "FAQ - Operation of a Fusor." January 2011.

[14] S Yu Gus'kov and Yu K Kurilenkov, Neutron yield and Lawson criterion for plasma with inertial electrostatic confinement, Journal of Physics: Conference Series 774 (2016) 012132. 